\title{
Theoretical study of 2-phenylpyrrole molecule using various quantum-chemical approaches
}

\author{
Lenka Rottmannováa, Kraiwan Punyain ${ }^{\mathrm{b}, \mathrm{c}}$, Ján Rimarčík \\ Vladimír Lukeša ${ }^{\mathrm{a}}$ Erik Klein ${ }^{\mathrm{a}}$, Anne-Marie Kelterer ${ }^{\mathrm{b}}$ \\ ${ }^{a}$ Institute of Physical Chemistry and Chemical Physics, Slovak University of Technology in Bratislava, \\ Radlinského 9, SK-812 37 Bratislava, Slovak Republic \\ ${ }^{b}$ Institute of Physical and Theoretical Chemistry, Graz University of Technology, \\ Stremayrgasse 9/I, A-8010 Graz, Austria \\ ${ }^{c}$ Department of Chemistry, Faculty of Science, Naresuan University, Phitsanulok 65000, Thailand \\ lenka.rottmannova@stuba.sk
}

\begin{abstract}
A systematic theoretical study of 2-phenylpyrrole (PhPy) is presented for its neutral and monocharged states. The calculations were performed using the semiempirical Austin Model 1 (AM1) method, $a b$ initio Møller-Plesset perturbation theory up to the second-order (MP2), density functional theory (DFT) and its tight-binding approximation (DFTB + ). The comparison of the obtained equilibrium geometries showed that the $\mathrm{C}-\mathrm{C}$ bond lengths in the phenylene ring are practically identical for the neutral state. Electric charging leads to significant changes in the geometry with respect to the neutral state. The $\mathrm{C}-\mathrm{N}$ bonds in PhPy are elongated and the negative charging produces the out-of-plane distortion of $\mathrm{N}-\mathrm{H}$ bond from the aromatic ring plane. The anionic state of the investigated molecule is connected with a higher perturbation of bond length alternation in both rings in comparison to the cationic state. The vibrationaly broadened absorption spectra, based on the on-the-fly molecular dynamics (MD) simulations, are also presented and compared with experimental spectra. Although the DFTB + method has the tendency to planarize the investigated molecular structure, the agreement of simulated absorption spectra based on the MD DFTB + geometries with TD-DFT calculations is acceptable.
\end{abstract}

Keywords: DFT, electro-optical properties, charged states, MP2, absorption spectra simulations

\section{Introduction}

Organic molecules with an extended aromatic structure have received great attention due to their application in electronic devices including organic light-emitting diodes (OLED), photosensitizers for photodynamic processes, field-effect transistors (FET) or various sensors (Cornil et al., 1998).

These molecules are very often built from simple five- and six-membered aromatic chromophoric units. These building blocks ensure the relatively well-defined molecular structure and chain lengths that allow the determination of structure-property relationships in detail. Therefore, a considerable effort is focused on the experimental and theoretical investigation of the electronic structure and photophysical properties of simple model chromophoric dimers, trimers or tetramers in neutral and charged states (Rubio et al., 2001). Although the combination of pyrrole $(\mathrm{Py})$ and phenylene $(\mathrm{Ph})$ units represents the basic molecular building unit in numerous oligomeric or star-burst systems with potential application in optoelectronics, the complex theoretical analysis oriented on the description of optimal geometries and electronic structures of neutral and monocharged states of the simple molecule is still missing. Therefore the main aim of this study is to employ DFT (Parr et al., 1991) and ab initio MP2 (Head-Gordon et al., 1988) methods for the calculation of the optimal geometries for the neutral and selected charged electronic groundstates of 2-phenylpyrrole (Fig. 1).

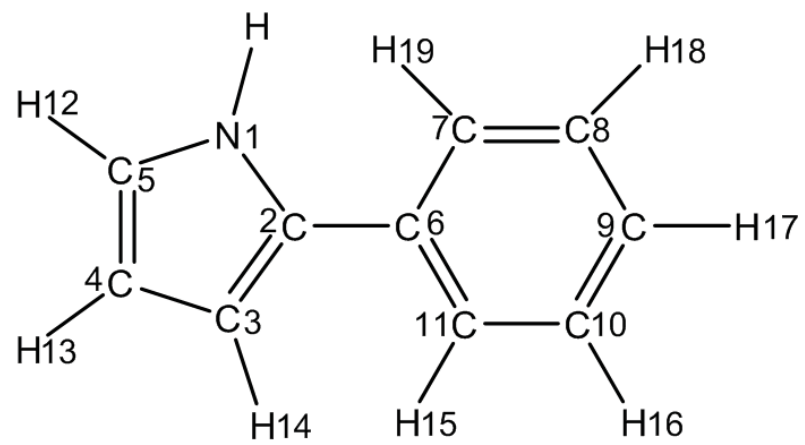

Fig. 1. Atom numbering for studied molecules. Dihedral angles are $\Theta=\Varangle(\mathrm{N} 1-\mathrm{C} 2-\mathrm{C} 6-\mathrm{C} 7)$

(Ph-Py rotation angle) and $\Lambda=\Varangle(\mathrm{C} 5-\mathrm{N} 1-\mathrm{H}-\mathrm{C} 2)$ $(\mathrm{N}-\mathrm{H}$ out of plane angle).

In this context, the quality and applicability of the semiempirical AM1 (Dewar et al., 1985) and DFTB+ (Aradi et al., 2007) quantum chemical treatments will be also discussed. Vertical excitation energies will be 
investigated with respect to their nature including the discussion of molecular orbitals. The influence of the neutral electronic ground-state torsional potential on the molecular motion and structural changes were determined by the application of the on-the-fly molecular dynamics (MD). Convolution of absorption spectra was performed for the obtained set of MD geometries using the time dependent (TD-)DFT theory (Furche et al., 2002) and CIS (Configuration Interaction Singles) method (Stratmann et al., 1998) based on the semiempirical ZINDO (Zerner's spectroscopic parameterization for intermediate neglect of differential overlap) Hamiltonian.

\section{Computational methods}

The optimal molecular geometries and torsional potentials of the studied molecule (Fig. 1) were calculated using DFT method with B3LYP (Becke's three parameter Lee-Yang-Parr) (Becke, 1996) and PBE (Perdew-Burke-Ernzerhof) (Perdew et al., 1996) functionals, as well as with the semiempirical AM1 and DFTB + methods. MP2 calculations have been performed as a benchmark. The torsional potential was calculated around the dihedral angle $\Theta$ with two interlinkage dihedral angles $\mathrm{N} 1-\mathrm{C} 2-\mathrm{C} 6-\mathrm{C} 7$ fixed for bonds N1-C2-C6-C7 and N1-C2 C6-C11 (Fig. 1), during optimization. Found optimal geometries were confirmed to be real minima by frequency calculation (no imaginary vibrations). On the basis of global minimum geometries, the vertical electronic excitation energies were calculated using the time-dependent DFT (TD-B3LYP) and semiempirical ZINDO approaches.
Classical MD simulations utilizing the gradients based on the above mentioned quantum chemical methods have been carried out with the Newton-X code (Barbatti et al., 2006). The Velocity-Verlet scheme was used to integrate the equations of motion. Using $0.25 \mathrm{fs}$ time steps, the dynamics was equilibrated for $20 \mathrm{ps}$ and then it has been running for further 25 ps. Temperature $(300 \mathrm{~K})$ was controlled by the Andersen thermostat. Absorption bands in the gas phase have been simulated by sampling the configuration space with 10000 points picked from the thermally equilibrated MD geometries. For these samples, 25 vertical electronic excitation energies and oscillator strengths between initial and final states were calculated by the TD-B3LYP and ZINDO methods. These quantities enabled the computation of Einstein coefficient $B$. The energy of every transition was smoothed by a Gaussian function with the height corresponding to Einstein coefficient and the width corresponding to arbitrary phenomenological broadening constant $(0.08 \mathrm{eV})$. The sum of all Gaussian functions plotted against the transition energy gives a post-Condon semi-classical approximation of the real spectrum.

The cc-pVDZ (Gill et al., 1992) basis set was used in the DFT and ab initio calculations. The calculations of optimal geometries and gradients were performed using ORCA (MP2 and B3LYP) (Neese et al., 2010), Mopac7 (AM1) (Stewart, 1993) and $\mathrm{DFTB}^{+}$program packages. The $\mathrm{DFTB}^{+}$calculations of optimal geometries for fixed dihedral angles were performed using the EFX code with implemented DFTB+ gradients (Baker, 1985). All

Tab. 1. Optimal bond lengths (in $\AA$ ), BLA values (in $\AA$ ) and dihedral angles $\Theta$ and $\Lambda$ (in deg) for the optimal geometries of PhPy molecule in neutral (0), cationic (+1) and anionic (-1) states.

\begin{tabular}{lcccccccccccccccc}
\hline Bond & \multicolumn{1}{c}{ MP2/cc-pVDZ } & \multicolumn{3}{c}{ B3LYP/cC-pVDZ } & \multicolumn{3}{c}{ PBE/cc-pVDZ } & \multicolumn{3}{c}{ DFTB+ } & \multicolumn{4}{c}{ AM1 } \\
\hline & 0 & +1 & -1 & 0 & +1 & -1 & 0 & +1 & -1 & 0 & +1 & -1 & 0 & +1 & -1 \\
\hline C4-C5 & 1.397 & 1.431 & 1.371 & 1.384 & 1.417 & 1.386 & 1.379 & 1.413 & 1.379 & 1.393 & 1.420 & 1.393 & 1.404 & 1.447 & 1.396 \\
C3-C4 & 1.423 & 1.388 & 1.431 & 1.423 & 1.383 & 1.422 & 1.417 & 1.377 & 1.417 & 1.414 & 1.387 & 1.416 & 1.430 & 1.389 & 1.426 \\
C2-C3 & 1.404 & 1.429 & 1.409 & 1.393 & 1.438 & 1.420 & 1.388 & 1.434 & 1.415 & 1.402 & 1.434 & 1.425 & 1.411 & 1.466 & 1.427 \\
C2-C6 & 1.466 & 1.428 & 1.456 & 1.465 & 1.428 & 1.425 & 1.460 & 1.423 & 1.421 & 1.459 & 1.431 & 1.425 & 1.450 & 1.417 & 1.414 \\
C6-C7 & 1.415 & 1.410 & 1.457 & 1.410 & 1.426 & 1.450 & 1.405 & 1.420 & 1.445 & 1.410 & 1.425 & 1.448 & 1.403 & 1.418 & 1.426 \\
C7-C8 & 1.404 & 1.364 & 1.397 & 1.396 & 1.384 & 1.386 & 1.392 & 1.380 & 1.381 & 1.395 & 1.386 & 1.382 & 1.393 & 1.386 & 1.380 \\
C8-C9 & 1.405 & 1.395 & 1.389 & 1.398 & 1.408 & 1.416 & 1.393 & 1.403 & 1.412 & 1.398 & 1.406 & 1.418 & 1.394 & 1.401 & 1.400 \\
C9-C10 & 1.407 & 1.390 & 1.462 & 1.400 & 1.405 & 1.425 & 1.395 & 1.400 & 1.421 & 1.399 & 1.405 & 1.424 & 1.395 & 1.398 & 1.405 \\
C10-C11 & 1.403 & 1.367 & 1.395 & 1.395 & 1.386 & 1.382 & 1.390 & 1.382 & 1.377 & 1.394 & 1.387 & 1.380 & 1.392 & 1.388 & 1.376 \\
C6-C11 & 1.414 & 1.411 & 1.420 & 1.410 & 1.427 & 1.445 & 1.405 & 1.421 & 1.439 & 1.410 & 1.426 & 1.444 & 1.404 & 1.419 & 1.428 \\
\hline BLA, Ph & 0.004 & 0.016 & 0.026 & 0.006 & 0.014 & 0.023 & 0.006 & 0.014 & 0.023 & 0.006 & 0.013 & 0.023 & 0.004 & 0.011 & 0.017 \\
BLA, Py & 0.014 & 0.027 & 0.022 & 0.013 & 0.025 & 0.011 & 0.014 & 0.027 & 0.012 & 0.013 & 0.024 & 0.009 & 0.011 & 0.032 & 0.010 \\
\hline N1-C5 & 1.375 & 1.342 & 1.384 & 1.373 & 1.355 & 1.405 & 1.365 & 1.349 & 1.393 & 1.371 & 1.355 & 1.406 & 1.387 & 1.370 & 1.413 \\
N1-C2 & 1.381 & 1.404 & 1.368 & 1.383 & 1.387 & 1.413 & 1.374 & 1.378 & 1.400 & 1.380 & 1.391 & 1.405 & 1.401 & 1.411 & 1.423 \\
N1-H & 1.014 & 1.016 & 1.015 & 1.011 & 1.014 & 1.015 & 1.009 & 1.012 & 1.011 & 1.034 & 1.037 & 1.036 & 0.985 & 0.995 & 0.991 \\
\hline$\Theta$ & 31 & 0 & 17 & 24 & 0 & 6 & 24 & 0 & 6 & 3 & 0 & 0 & 26 & 18 & 19 \\
$\Lambda$ & 8 & 0 & 9 & 8 & 0 & 34 & 8 & 0 & 30 & 1 & 0 & 29 & 2 & 2 & 40 \\
\hline
\end{tabular}


calculations of optical transitions (TD-B3LYP and ZINDO) were done using the Gaussian 03 package (Frisch et al., 2003).

\section{Results and discussion}

\section{Structure description of neutral and charged states}

Found theoretical bond lengths for the studied molecules are compiled in Tables 1 and 2. In the case of the neutral PhPy molecule, all used methods give the minimal bond lengths for the bond $\mathrm{N} 1-\mathrm{C} 5$ and N1-C2 which are between the Py nitrogen and carbon atoms (see Fig. 1). The smallest B3LYP, PBE and DFTB+ values are $1.373 \AA$, $1.365 \AA$ and $1.371 \AA$, respectively. The reference MP2 method gives also the minimal value of 1.375 $\AA$ for the bond N1-C5. The differences between semiempirical AM1 bond lengths and the reference MP2 are $0.012 \AA$ for the bond N1- C5 and $0.020 \AA$ for the bond N1-C2.

Electron abstraction or addition leads to a perturbation of the aromatic structure. It seems that the structural changes are distributed over the whole molecular skeleton. In the case of the optimal B3LYP and PBE geometries, the negative charging is responsible for the elongation of bonds connecting the heteroatoms with carbon atoms (N1-C5 and $\mathrm{N} 1-\mathrm{C} 2)$ or the C2-C3, C6-C7, C8-C9, C9-C10 and C6-C11 bonds. Significant shortening was found for the bonds C2-C6, C7-C8 and C10-C11. The used quantum chemical treatments for the anionic state also showed that the hydrogen atom connected with the nitrogen atom is significantly distorted from the plane of the pyrrole ring compared to the neutral state (see the N1-H bond in Table 1). The out-of-plane distortion for the anionic state is $9^{\circ}$ for $\mathrm{MP} 2,29^{\circ}$ for $\mathrm{DFTB}+, 30^{\circ}$ for $\mathrm{PBE}, 34^{\circ}$ for B3LYP and $40^{\circ}$ for AM 1 (see angle $\Lambda$ in Table 1 ). The planarization of the molecule aromatic fragments (B3LYP, DFTB+, PBE) leads to a repulsion between the hydrogen atom at nitrogen (atom 20) and the interacting hydrogen atom at the phenylene ring (atom 19). Therefore this hydrogen atom moves out of plane. A planar $\mathrm{H}-\mathrm{N}$ pyrrole conformation would have about $1.67 \mathrm{~kJ} \mathrm{~mol}^{-1}$ higher relative energy (B3LYP).

The data collected in Table 1 for the cationic states indicate that the positive charging has a more uniform influence on the bond lengths than the negative charging in the anion. The bonds $\mathrm{N} 1-\mathrm{C} 5$, C3 - C4 and C2-C6 are shortened while the bonds N1-C2, C4-C5, C2-C3 and C6-C7 are elongated. In order to characterize the alternation of single or double carbon-carbon bonds within the two ring moieties, the Bond Length Alternation parameter
(BLA) can be used (Jacquemin et al., 2007). This parameter might be defined as the average sum of absolute values of the differences between the $i$-th bond length $d_{i}$ and average bond length $\bar{d}$.

$$
B L A=\frac{\sum_{i}\left|d_{i}-\bar{d}\right|}{N}
$$

In Equation (1), $N$ stands for the number of conjugated $\mathrm{C}-\mathrm{C}$ bonds. In our case, the average bond length $\bar{d}$ is obtained for the phenylene ring and $N=6$. For the Py ring, the summation in Equation (1) runs over the bonds N1-C2, C2-C3, C3-C4, C4-C5, C5-N1 with $N=5$. With respect to this definition, a very small $B L A$ value indicates an effective aromatic structure. In the case of the isolated benzene molecule this value equals zero. As it can be seen from data in Table 1, both neutral molecules have the lowest $B L A$. These values lie between 0.004 and $0.006 \AA$ for the phenylene ring and from 0.009 to $0.032 \AA$ for the pyrrole ring. Significant increase of the $B L A$ in the $\mathrm{Ph}$ subunit (more than $0.017 \AA$ ) with respect to the neutral state occurs in the anionic structure. The mutual comparison of this parameter for the studied molecule shows that the MP2, DFT and DFTB+ values are larger by about $25 \%$ for the negatively charged molecule. Charging the $\mathrm{PhPy}$ molecule induces the largest bond length changes in the pyrrole ring for the cationic state. The Py BLA value is $0.027 \AA$ for MP2, $0.025 \AA$ for B3LYP, and $0.032 \AA$ for AM1 methods, respectively. An even smaller Py BLA occurs upon negative charging in the pyrrole ring, but it is still smaller than in the Ph ring.

\section{Energy barriers and torsional potentials for neutral and charged states}

The mutual orientation of aromatic rings represents the key factor which modifies the electron $\pi$-conjugation. The computed dihedral angles for optimal structures are collected in Table 1.

The torsional angles $\Theta$ calculated for the neutral state of PhPy molecule using MP2, DFT and AM1 methods exhibit the values in the range from 24 to 35 degrees. The semiempirical AM1 method, parameterized for aromatic molecules, gives the optimal dihedral angle for PhPy of $26^{\circ}$. These results are smaller by $5^{\circ}$ and $11^{\circ}$ in comparison to the reference $a b$ initio MP2 calculations. The B3LYP/ cc-pVDZ and PBE/cc-pVDZ torsional angles are qualitatively comparable with the MP2 and AM1 results. In contrast, the $\mathrm{DFTB}+$ method gives practically planar structures. This might be explained as the consequence of the applied tight-binding approximation. The dependence of the relative energy on dihedral angles for used computational 


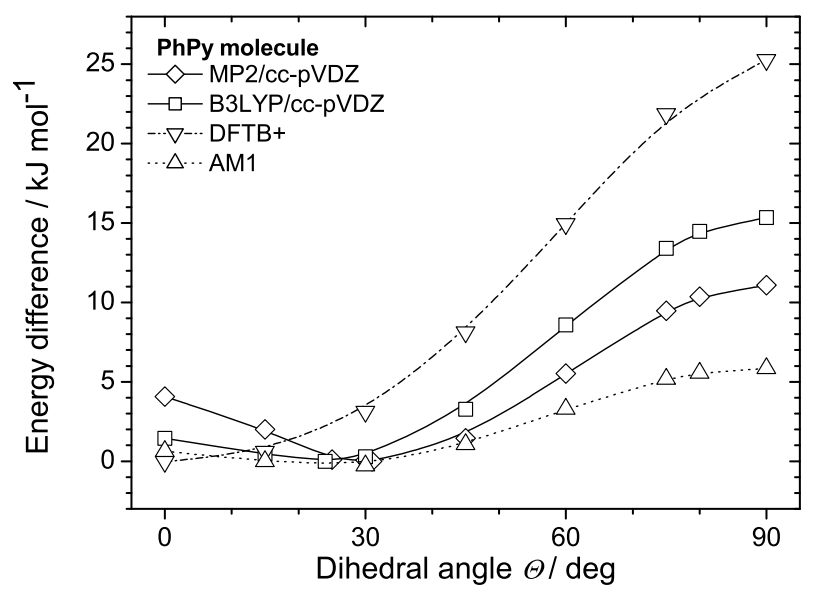

a)

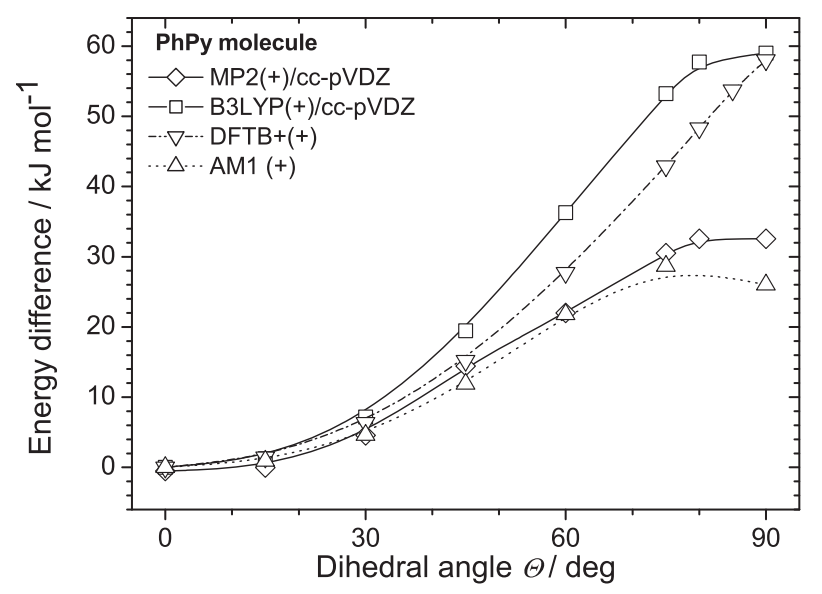

b)

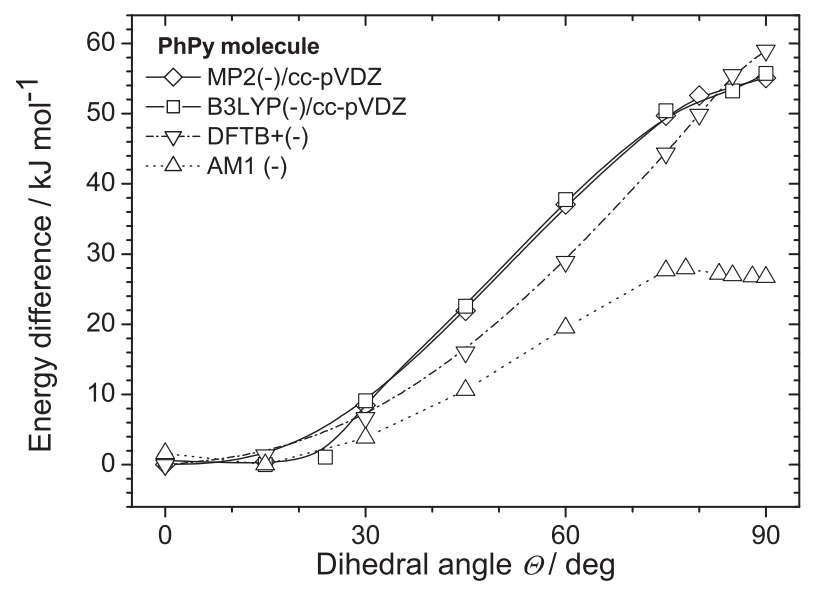

c)

Fig. 2. Dependence of the relative energy on the dihedral angle $\Theta$ for the a) neutral; b) cationic (+1) and c) anionic (-1) states of PhPy calculated at the MP2/cc-pVDZ, B3LYP/cc-pVDZ, DFTB+ and AM1 levels of theory. The respective ground state energy minimum represents the reference value. methods in the case of the neutral state is given in Fig. 2.

This comparison represents a critical test, since the correct description of torsional motions is crucial for the subsequent dynamics simulations. The calculated curves for the neutral state exhibit one minimum for non-planar structures and two saddle points for planar and perpendicular arrangements. The corresponding energies of the located barriers are collected in Table 2.

All used quantum chemical methods showed that the PhPy molecule has higher relative energies for the perpendicular arrangement of the rings. For example, the calculated MP2 energy barrier of the perpendicular $\mathrm{PhPy}$ reached $11.10 \mathrm{~kJ} \mathrm{~mol}^{-1}$. The mutual comparison of these rotational energies with respect to the used methods shows that the semiempirical DFTB + method overestimates the energy barrier by a factor of more than 2.2 with respect to the MP2 energies. The AM1 method underestimates the PhPy barrier. This is connected with the fact that the AM1 method does not describe properly the pyramidalization and rotation barriers for amino groups (Jensen, 2007).

The geometrical changes with respect to positive or negative charging correlate with the interring dihedral angle changes. The shortening of C2-C6 bond occurs with the tendency of the molecular skeleton planarization (see Tables 1 and 2) with decreasing $B L A$ to the neighbouring bonds as an indication of an increasing aromaticity, especially in the cationic state. All used quantum chemical methods provide a fully planar cationic state. As presented in Fig. 2, the DFTB + curves have one minimum for the planar orientation and one saddle point for the perpendicular arrangement. A very flat minimum for the perpendicular arrangement is computed for the positively and negatively charged (AM1) and anionic (MP2, B3LYP) states of PhPy.

\section{Electronic structure and absorption spectra simulation}

The presence of the $\mathrm{N}-\mathrm{H}$ group in the heterocyclic ring influences the excited electronic states. The first six vertical excitation energies are summarized in Table 3. Only one transition results in a computed oscillator strength higher than 0.5, while the other transitions with smaller oscillator strengths have a negligible relevance for the experiment in solution. In the gas phase calculations, the TD-B3LYP transition based on the optimal MP2/cc-pVDZ and B3LYP/cc-pVDZ geometries with significant oscillator strengths belongs to the $\mathrm{S}_{0} \rightarrow \mathrm{S}_{2}$ transition. The comparison of the energies between $S_{0} \rightarrow S_{1}$ and $S_{0} \rightarrow S_{2}$ transitions indicate small differences $(0.04$ to $0.05 \mathrm{eV})$ for the $\mathrm{PhPy}$ 
Tab. 2. Energy barriers (in $\mathrm{kJ} \mathrm{mol}^{-1}$ ) with respect to the global minimum for PhPy in neutral (0), cationic $(+1)$ and anionic $(-1)$ states. The values of dihedral angle $\Theta$ (in deg) for near perpendicular arrangement are indicated in parentheses.

\begin{tabular}{lcccccc}
\hline Method & \multicolumn{5}{c}{$\begin{array}{c}\text { Energy barrier / } \mathrm{kJ} \mathrm{mol}^{-1} \\
\text { perpendicular or near perpendicular structure }\end{array}$} \\
\hline & & & planar structure & \multicolumn{5}{c}{+1} \\
PhPy: & & +1 & -1 & 0 & & +1 \\
MP2/cc-pVDZ & 4.06 & - & 0.70 & 11.10 & 32.54 & 55.08 \\
B3LYP/cc-pVDZ & 1.44 & - & 0.66 & 15.37 & 59.00 & 55.72 \\
PBE/cc-pVDZ & 1.60 & - & 0.60 & 15.30 & 53.90 & 54.60 \\
DFTB+ & 0.03 & - & 0.03 & 25.29 & 58.00 & 59.03 \\
AM1 & 0.89 & - & 1.62 & 5.62 & $28.07\left(77^{\circ}\right)$ & $27.50\left(78^{\circ}\right)$ \\
\hline
\end{tabular}

Tab. 3. Theoretical excitation energies (in $\mathrm{eV}$ ) and oscillator strengths (in parentheses) for the six lowest electronic excitations for the optimal neutral PhPy geometry.

\begin{tabular}{|c|c|c|c|c|c|c|}
\hline & $S_{0} \rightarrow S_{1}$ & $\mathbf{S}_{0} \rightarrow \mathbf{S}_{2}$ & $\mathbf{S}_{0} \rightarrow \mathbf{S}_{3}$ & $\mathbf{S}_{0} \rightarrow \mathbf{S}_{4}$ & $\mathbf{S}_{0} \rightarrow \mathbf{S}_{5}$ & $\mathbf{S}_{0} \rightarrow \mathbf{S}_{6}$ \\
\hline TD-B3LYP//MP2/cc-pVDZ & $\begin{array}{c}4.35 \\
(0.012)\end{array}$ & $\begin{array}{c}4.41 \\
(0.432)\end{array}$ & $\begin{array}{c}5.39 \\
(0.022)\end{array}$ & $\begin{array}{c}5.53 \\
(0.022)\end{array}$ & $\begin{array}{c}5.71 \\
(0.003)\end{array}$ & $\begin{array}{c}5.77 \\
(0.003)\end{array}$ \\
\hline TD-B3LYP//B3LYP/cc-pVDZ & $\begin{array}{c}4.41 \\
(0.007)\end{array}$ & $\begin{array}{c}4.44 \\
(0.480)\end{array}$ & $\begin{array}{c}5.40 \\
(0.006)\end{array}$ & $\begin{array}{c}5.60 \\
(0.030)\end{array}$ & $\begin{array}{c}5.74 \\
(0.003)\end{array}$ & $\begin{array}{c}5.78 \\
(0.011)\end{array}$ \\
\hline TD-B3LYP//PBE/cc-pVDZ & $\begin{array}{c}4.57 \\
(0.489)\end{array}$ & $\begin{array}{c}4.58 \\
(0.015)\end{array}$ & $\begin{array}{c}5.62 \\
(0.005)\end{array}$ & $\begin{array}{c}5.77 \\
(0.037)\end{array}$ & $\begin{array}{c}6.06 \\
(0.003)\end{array}$ & $\begin{array}{c}6.09 \\
(0.010)\end{array}$ \\
\hline TD-B3LYP/cc-pVDZ//DFTB+ & $\begin{array}{c}4.35 \\
(0.282)\end{array}$ & $\begin{array}{c}4.37 \\
(0.260)\end{array}$ & $\begin{array}{c}5.31 \\
(0.000)\end{array}$ & $\begin{array}{c}5.39 \\
(0.003)\end{array}$ & $\begin{array}{c}5.62 \\
(0.040)\end{array}$ & $\begin{array}{c}5.88 \\
(0.039)\end{array}$ \\
\hline TD-B3LYP/cc-pVDZ//AM1 & $\begin{array}{c}4.35 \\
(0.011)\end{array}$ & $\begin{array}{c}4.39 \\
(0.391)\end{array}$ & $\begin{array}{c}5.40 \\
(0.007)\end{array}$ & $\begin{array}{c}5.60 \\
(0.029)\end{array}$ & $\begin{array}{c}5.69 \\
(0.002)\end{array}$ & $\begin{array}{c}5.81 \\
(0.013)\end{array}$ \\
\hline TD-B3LYP(ethanol)//MP2/cc-pVDZ & $\begin{array}{c}4.33 \\
(0.536)\end{array}$ & $\begin{array}{c}4.38 \\
(0.014)\end{array}$ & $\begin{array}{c}5.40 \\
(0.007)\end{array}$ & $\begin{array}{c}5.54 \\
(0.036)\end{array}$ & $\begin{array}{c}5.74 \\
(0.004)\end{array}$ & $\begin{array}{c}6.02 \\
(0.031)\end{array}$ \\
\hline TD-B3LYP(ethanol)//B3LYP/cc-pVDZ & $\begin{array}{c}4.36 \\
(0.579)\end{array}$ & $\begin{array}{c}4.44 \\
(0.016)\end{array}$ & $\begin{array}{c}5.40 \\
(0.006)\end{array}$ & $\begin{array}{c}5.60 \\
(0.049)\end{array}$ & $\begin{array}{c}5.80 \\
(0.015)\end{array}$ & $\begin{array}{c}6.10 \\
(0.019)\end{array}$ \\
\hline TD-PBE(ethanol)//PBE/cc-pVDZ & $\begin{array}{c}3.86 \\
(0.001)\end{array}$ & $\begin{array}{c}4.00 \\
(0.531)\end{array}$ & $\begin{array}{c}4.71 \\
(0.007)\end{array}$ & $\begin{array}{c}4.83 \\
(0.017)\end{array}$ & $\begin{array}{c}5.19 \\
(0.028)\end{array}$ & $\begin{array}{c}5.56 \\
(0.108)\end{array}$ \\
\hline TD-B3LYP(ethanol)/cc-pVDZ//DFTB+ & $\begin{array}{c}4.25 \\
(0.624)\end{array}$ & $\begin{array}{c}4.38 \\
(0.032)\end{array}$ & $\begin{array}{c}5.38 \\
(0.002)\end{array}$ & $\begin{array}{c}5.61 \\
(0.061)\end{array}$ & $\begin{array}{c}5.86 \\
(0.049)\end{array}$ & $\begin{array}{c}6.05 \\
(0.000)\end{array}$ \\
\hline TD-B3LYP(ethanol)/cc-pVDZ//AM1 & $\begin{array}{c}4.29 \\
(0.554) \\
\end{array}$ & $\begin{array}{c}4.39 \\
(0.041) \\
\end{array}$ & $\begin{array}{c}5.40 \\
(0.006) \\
\end{array}$ & $\begin{array}{c}5.61 \\
(0.046) \\
\end{array}$ & $\begin{array}{c}5.83 \\
(0.016) \\
\end{array}$ & $\begin{array}{c}6.04 \\
(0.062) \\
\end{array}$ \\
\hline ZINDO//MP2/cc-pVDZ & $\begin{array}{c}3.91 \\
(0.565)\end{array}$ & $\begin{array}{c}4.25 \\
(0.017)\end{array}$ & $\begin{array}{c}4.88 \\
(0.032)\end{array}$ & $\begin{array}{c}5.15 \\
(0.006)\end{array}$ & $\begin{array}{c}5.33 \\
(0.123)\end{array}$ & $\begin{array}{c}5.42 \\
(0.084)\end{array}$ \\
\hline ZINDO//B3LYP/cc-pVDZ & $\begin{array}{c}3.92 \\
(0.587)\end{array}$ & $\begin{array}{c}4.28 \\
(0.013)\end{array}$ & $\begin{array}{c}4.94 \\
(0.028)\end{array}$ & $\begin{array}{c}5.20 \\
(0.007)\end{array}$ & $\begin{array}{c}5.38 \\
(0.116)\end{array}$ & $\begin{array}{c}5.46 \\
(0.111)\end{array}$ \\
\hline ZINDO//PBE/cc-pVDZ & $\begin{array}{c}3.94 \\
(0.585)\end{array}$ & $\begin{array}{c}4.30 \\
(0.014)\end{array}$ & $\begin{array}{c}4.96 \\
(0.029)\end{array}$ & $\begin{array}{c}5.23 \\
(0.007)\end{array}$ & $\begin{array}{c}5.42 \\
(0.139)\end{array}$ & $\begin{array}{c}5.50 \\
(0.085)\end{array}$ \\
\hline ZINDO//DFTB + & $\begin{array}{c}3.77 \\
(0.619)\end{array}$ & $\begin{array}{c}4.21 \\
(0.016)\end{array}$ & $\begin{array}{c}4.88 \\
(0.025)\end{array}$ & $\begin{array}{c}5.17 \\
(0.004)\end{array}$ & $\begin{array}{c}5.35 \\
(0.004)\end{array}$ & $\begin{array}{c}5.39 \\
(0.204)\end{array}$ \\
\hline ZINDO//AM1/cc-pVDZ & $\begin{array}{c}3.81 \\
(0.582) \\
\end{array}$ & $\begin{array}{c}4.26 \\
(0.016) \\
\end{array}$ & $\begin{array}{c}4.83 \\
(0.024) \\
\end{array}$ & $\begin{array}{c}5.13 \\
(0.007) \\
\end{array}$ & $\begin{array}{c}5.35 \\
(0.066) \\
\end{array}$ & $\begin{array}{c}5.42 \\
(0.130) \\
\end{array}$ \\
\hline
\end{tabular}

molecule. The available experimental value of absorption maximum in ethanol is $4.40 \mathrm{eV}$ (Kosak et al., 1954; Strashnikova et al., 1993), $4.32 \mathrm{eV}$ in acetonitrile (Turchaninov et al., 1997) and $4.23 \mathrm{eV}$ in dimethylsulfoxide (Murzina et al., 2002). The presented theoretical gas phase excitation energies with dominant oscillator strengths are by about $0.25 \mathrm{eV}$ higher. On the other hand, the inclusion of the solvent effect (for ethanol) using the cavity IEF-PCM (Polarizable Continuum Model using the Integral Equation Formalism variant) (Tomasi et al., 1999) method or semiempirical ZINDO Hamiltonian leads to an energy decrease by $0.1 \mathrm{eV}$ (and $0.3 \mathrm{eV}$ for PBE). Generally, the PBE functional gives higher vertical excitation energies compared to the B3LYP ones.

In order to understand the role of the $\mathrm{N}-\mathrm{H}$ group for the photon absorption, it is useful to examine the relevant (highest) occupied and lowest unoccupied molecular orbitals playing a dominant role within 

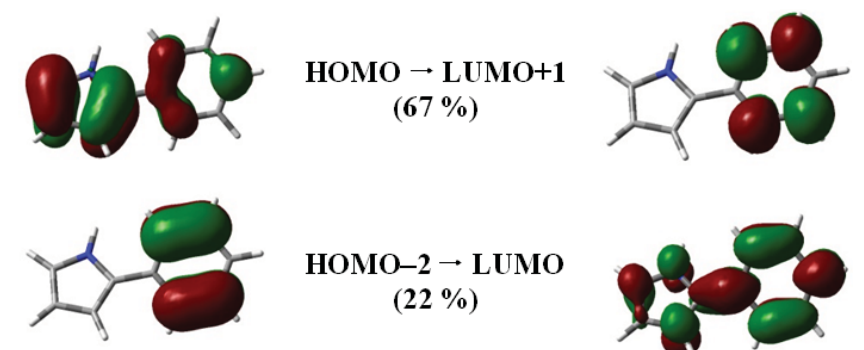

HOMO-2 $\rightarrow$ LUMO
$(22 \%)$

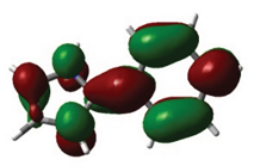

$4.44 \mathrm{eV}(0.4801)$

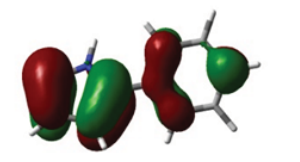

HOMO $\rightarrow$ LUMO
$(64 \%)$

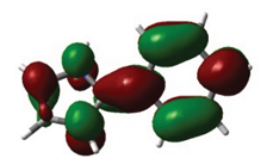

Fig. 3. Plots of the B3LYP/cc-pVDZ molecular orbitals significantly contributing to the TD-B3LYP lowest energy transitions of neutral PhPy molecule. The values in parentheses stand for percentages of the excitation contributions in individual transitions. The isosurface value is $0.015 \mathrm{Bohr}^{-3 / 2}$.

electron transitions. As it can be seen in Fig. 3, an electron is excited from the highest occupied molecular orbital (HOMO) to the lowest unoccupied molecular orbital (LUMO) within the first electronic excitation. The B3LYP/cc-pVDZ shows the HOMO of $\mathrm{PhPy}$ delocalized over the pyrrole ring between the $\mathrm{C} 2-\mathrm{C} 3$ and $\mathrm{C} 4-\mathrm{C} 5$ bonds. The corresponding LUMO in the strongest transition is delocalized mostly over the C2-C6, C7-C8 and C10-C11 bonds. In the case of the transitions with very small oscillator strength, the electronic excitation takes place from HOMO-2 to LUMO and from HOMO to $\mathrm{LUMO}+1$ orbitals. These orbitals are delocalized over the phenylene unit only. This transition may be connected with the electron transfer between the heterocyclic ring and the phenylene moiety.

The simulated MD absorption spectrum based on MD-B3LYP, MD-DFTB+ and MD-AM1 geometries is shown in Fig. 4 together with the available experimental spectra. First, the spectral lines obtained by the gas phase TD-B3LYP calculations are narrow in comparison with experiment and the bands are blue shifted. This reflects the omitting of the solvent effects in quantum chemical calculations. On the other hand, the simulations of spectra including solvent effects by proper parameterization (ZINDO Hamiltonian) or by the cavity model (IEF$\mathrm{PCM}=$ ethanol) lead to broader peaks.

However, the ZINDO bands are shifted to lower energies by more than $0.5 \mathrm{eV}$ compared to experiment. The quality of the simulation performed using the MD-DFTB+ geometries is comparable. The tendency for the planarization of molecule is responsible for the red shift of the simulated bands

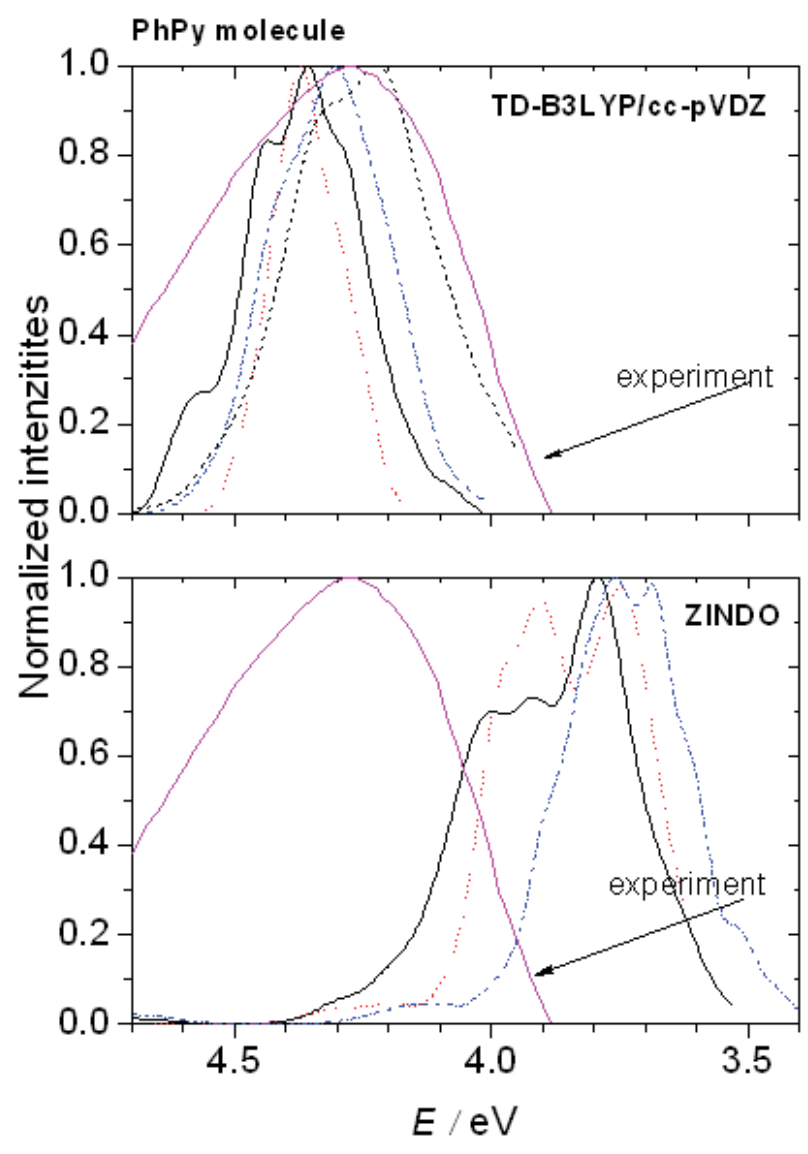

Fig. 4. Experimental (Turchaninov et al. (1997)) and simulated absorption spectra for neutral PhPy molecule based on TD-B3LYP/cc-pVDZ and ZINDO excitation energies (solid lines: MD-B3LYP, dotted lines: MD-AM1, dash-dotted lines: MD-DFTB + geometries, respectively). The dashed lines stand for the TD-B3LYP(ethanol)// MD-B3LYP/cc-pVDZ simulations. 
with respect to other simulations based on B3LYP and AM1 geometries.

The evaluated spectral characteristics may be related to the fluctuation of the torsional angle $\Theta$ and bond length alternation $(B L A)$. The distributions of the torsional angle are plotted in Fig. 5a for all three used quantum chemical methods. The position of the mean value reflects also the shape of torsional potentials presented in Fig. 2. DFTB + method gives the highest energy barrier for the perpendicular arrangement. Therefore, the average value of $\Theta$ corresponds to planar structures. On the other hand, the decrease in the barrier height is connected with the opposite effect shifting the probability of geometry to higher $\Theta$ angles. For example, the MD-AM1 method exhibits the most probable distortion at 45 degree while the population of a planar structure is less probable. This is consistent with the lowest barrier at $\Theta=90$ degrees (see Fig. 2). Additionally, the distribution of $B L A$ values is different from the static calculations. As it can be seen from Fig. $5 \mathrm{~b}$, the $\mathrm{Ph} B L A$ values for the phenylene moiety obtained from the MD geometries at $T=300 \mathrm{~K}$ are shifted to higher values in comparison with the optimal static geometry. For example, the positions of probability maximum are changed from $0.006 \AA$ (B3LYP/cc-pVDZ) to $0.035 \AA$ for MD-B3LYP/cc-pVDZ.

\section{Conclusions}

In this paper, the electrically neutral and monocharged states of 2-phenylpyrrole were studied using MP2, DFT, DFTB+ and AM1 methods. Based on these calculations, we have quantified the electronic structure between the six- and five-membered aromatic rings where the heteroatom is represented by a small nitrogen. The mutual comparison of the obtained equilibrium geometries showed that the $\mathrm{C}-\mathrm{C}$ bond lengths on phenylene rings are practically identical for the neutral molecule. On the other hand, electric charging leads to significant changes in the optimal geometries with respect to the neutral state of the studied model molecule. For example, the $\mathrm{C}-\mathrm{N}$ bonds are elongated. Negative charging is also responsible for the out-of-aromatic-plane distortion of $\mathrm{N}-\mathrm{H}$ bond which can be explained by decreasing aromaticity. If we compare the PES-cut for the torsional motion around the interfragment bond, the position of the global minimum and the rotational barrier heights are changed due to charging. For the neutral states, the highest barrier is connected with the perpendicular orientation and the lowest barrier with the planar arrangement. In the case of the charged states, the aromatic bond length distribution is disturbed and the stable molecular geometries are planar. Comparing with the neutral
PhPy molecule
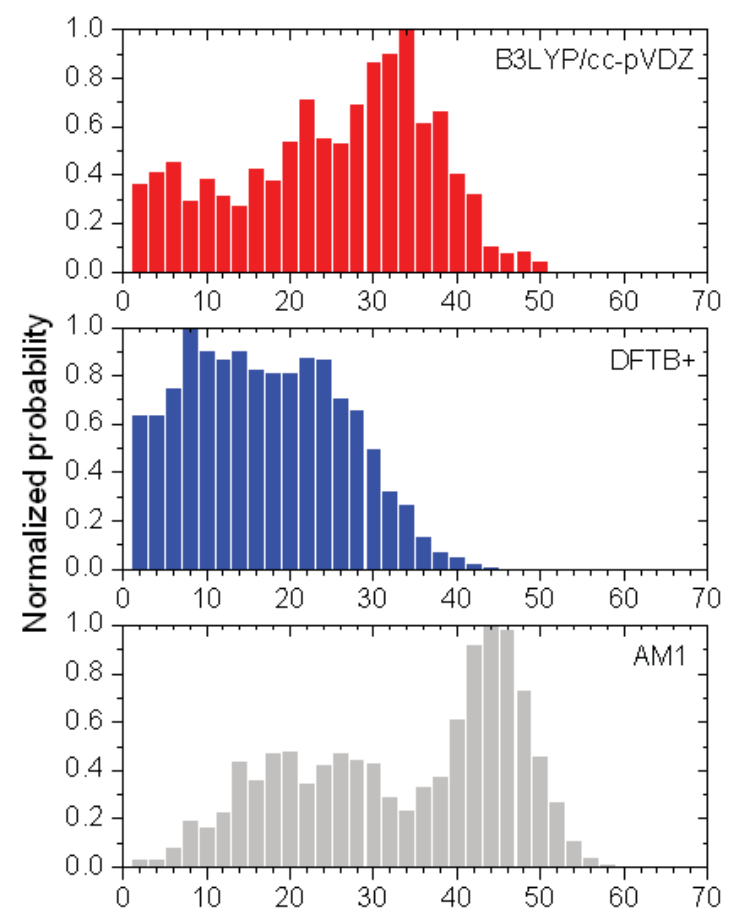

a) Dihedral angle $\Theta / \operatorname{deg}$

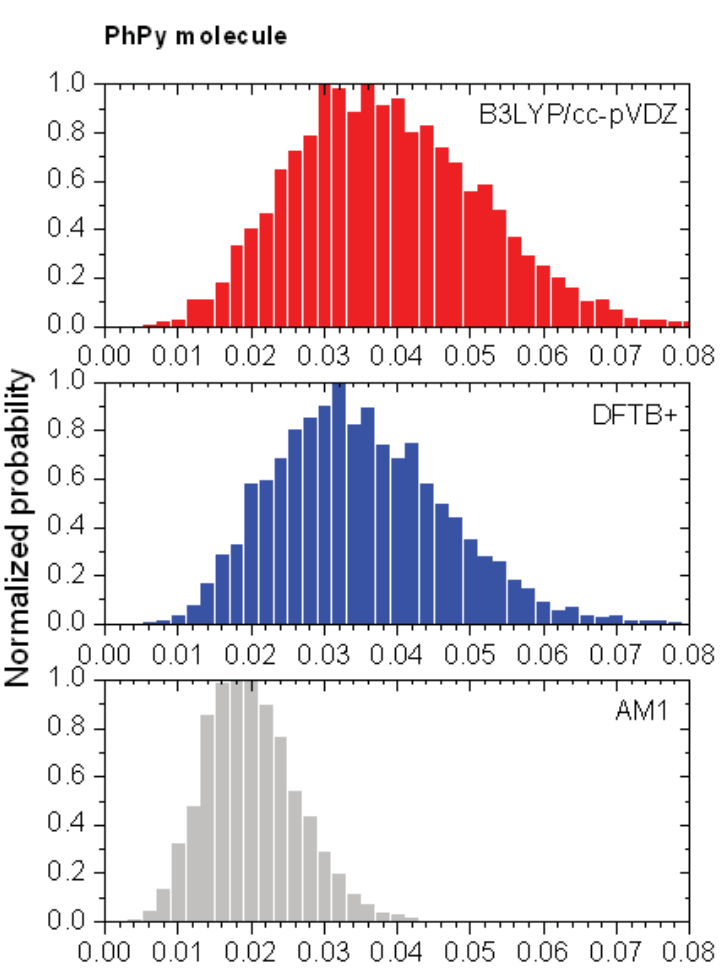

b) $B L A$ /angstrom

Fig. 5. Normalized absolute value distribution of the a) interring torsion angle $\Theta$ and b) phenylene BLA obtained from the MD simulations. 
states, the torsional barriers at $\Theta=90^{\circ}$ are twice as high for anions and three-times higher for cations. The simulations of absorption spectra were based on the combination of the semiclassical molecular dynamics and quantum chemical treatments. The calculations based on the TD-DFT(IEFPCM=ethanol) approach is in agreement with experiment showing that the excitation energies are slightly red shifted by about $0.10-0.20 \mathrm{eV}$. Although the $\mathrm{DFTB}^{+}$approach has the tendency to keep the structures planar, the obtained spectral bands are comparable with the AM1 and B3LYP/cc-pVDZ simulations. The inclusion of the solvent effect using the TDB3LYP(IEFPGM=ethanol) model or the calculations of the excitation energies using ZINDO Hamiltonian lead to a more realistic broadening of spectral bands. This broadening is comparable with experimental observations. However, ZINDO method gives unrealistic values for the excitation energies compared to the available experimental spectra. In this context, we can conclude that the combination of $\mathrm{DFTB}^{+}$geometries with TD-B3LYP(IEFPCM) excitation energies seems to be well suited for the spectral treatment of huge neutral molecules constructed from the class of conjugated system investigated in this work.

\section{Acknowledgement}

This work was supported by Slovak Grant Agency VEGA (1/1072/11) and the Science and Technology Assistance Agency (Projects No. SK-AT 0002-08 and SK-AT 0004-10), as well as a by the Austrian Academic Exchange Service program Wissenschaftlich-Technische Zusammenarbeit Austria-Slovakia under contracts Nos. ÖAD WTZ 11/2009 and 06/2011.

\section{References}

Cornil J, Beljonne D, Brédas JL (1998) Electronic Materials: The Oligomer Approach. Müllen and Wegner, Weinheim, New York (1998).
Rubio M, Ortí E, Pou-Amérigo R, Merchán M (2001) J. Phys. Chem. A. 105: 9788-9794.

Parr RG, Yang W, Springer-Verlag. 1991.

Head-Gordon MJ, Pople A, Frisch MJ (1988) Chem. Phys. Lett. 153: 503.

Dewar MJS, Zoebitsch EG, Healy EF, Stewart JJP (1985) J. Am. Chem. Soc. 107: 3902.

Furche F, Ahlrichs R. (2002) J. Chem. Phys. 117: 7433.

Stratmann RE, Scuseria GE, Frisch MJ (1998) J. Chem. Phys. 109: 8218.

Becke AD (1996) J. Chem. Phys. 104: 1040.

Perdew JP, Burke K, Ernzerhof M (1996) Phys. Rev. Lett. 77: 3865 .

Barbatti M, Granucci G, Persico M, Ruckenbauer M, Lischka H, Newton-X: a package for Newtonian dynamics close to the crossing seam, version $0.13 \mathrm{~b}$, www.univie.ac.at/newtonx, 2006.

Gill PMW, Johnson BG, Pople JA, Frisch MJ (1992) Chem. Phys. Lett. 197: 499.

Stewart JJP (1993) MOPAC 93.00 Manual Fujitsu Limited, Tokyo.

Aradi B, Hourahine B, Frauenheim T (2007) J. Phys. Chem. A, 111: 5678.

Baker J (1985) J. Comp. Chem. 7:385.

Frisch MJ, Trucks GW, Schlegel HB et al (2003) GAUSSIAN 03, Revision A.1, Gaussian, Inc., Pittsburgh, PA.

Jacquemin D, Perpete EA, Chermette H, Ciofini I, Adamo C (2007) Chem. Phys. 332: 79.

Jensen F (2007) Introduction to Computational Chemistry, Second edition, J. Wiley \& Sons Ltd., West Sussex (England).

Kosak AI, Palchak RJF, Steele WA, Selwitz ChM (1954) J. Am. Chem. Soc. 76: 4450.

Strashnikova NV, Sigalov MV, Korostova SE, Mikhaleva AE, Trofimov BA (1993) Russ. Chem. Bull. 42: 1013.

Turchaninov VK, Vokin AI, Korostova SE (1997) Russ. Chem. Bull. 46: 1407.

Murzina NM, Naimushina MI, Vokin AI, Tarasova OA, Mikhaleva AI, Turchaninov VK, Trofimov BA (2002) Russ. J. Gen. Chem. 72: 116.

Tomasi J, Mennucci B, Cancès E (1999) J. Mol. Struct. (Theochem) 464: 211.

Neese F, Becker U, Ganyushin D et al (2010) ORCA Version 2.8, An ab initio, DFT and semiempirical SCF-MO package, Bonn, Germany. 